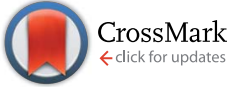

Cite this: Nanoscale, 2014, 6, 11810

\title{
Turbulence-assisted shear exfoliation of graphene using household detergent and a kitchen blender $\dagger$
}

\author{
Eswaraiah Varrla, ${ }^{\text {ac }}$ Keith R. Paton, ${ }^{\text {ab }}$ Claudia Backes, ${ }^{\text {ac }}$ Andrew Harvey, ${ }^{\text {ac }}$ \\ Ronan J. Smith, ${ }^{\text {ac }}$ Joe McCauley ${ }^{\text {ac }}$ and Jonathan N. Coleman ${ }^{\star a c}$
}

\begin{abstract}
To facilitate progression from the lab to commercial applications, it will be necessary to develop simple, scalable methods to produce high quality graphene. Here we demonstrate the production of large quantities of defect-free graphene using a kitchen blender and household detergent. We have characterised the scaling of both graphene concentration and production rate with the mixing parameters: mixing time, initial graphite concentration, rotor speed and liquid volume. We find the production rate to be invariant with mixing time and to increase strongly with mixing volume, results which are important for scale-up. Even in this simple system, concentrations of up to $1 \mathrm{mg} \mathrm{ml}^{-1}$ and graphene masses of $>500 \mathrm{mg}$ can be achieved after a few hours mixing. The maximum production rate was $\sim 0.15 \mathrm{~g} \mathrm{~h}^{-1}$, much higher than for standard sonication-based exfoliation methods. We demonstrate that graphene production occurs because the mean turbulent shear rate in the blender exceeds the critical shear rate for exfoliation.
\end{abstract}

Received 25th June 2014

Accepted 14th August 2014

DOI: $10.1039 / \mathrm{c} 4 \mathrm{nr} 03560 \mathrm{~g}$

www.rsc.org/nanoscale

Over the last decade, graphene has become one of the most studied of all nano-materials due to its 2-dimensional structure and its unique set of physical properties. ${ }^{1,2}$ During this period, the focus of much of the research community has been on mapping out and understanding the fundamental physics and chemistry of graphene. However, in recent years, the emphasis has started to shift slightly towards the demonstration of applications. ${ }^{3}$ Over the next few years, we expect the emphasis to shift further as both academic and industrial researchers concentrate on fulfilling the applications potential of graphene, eventually leading to a range of graphene-enabled products.

However, before this can be achieved, it will be critically important to develop industrially scalable production methods for graphene. While graphene can be produced by a range of techniques, many applications will require solution-processed ${ }^{4}$ graphene. In particular, a number of applications will require access to large volumes of graphene dispersions or inks. Using standard solution deposition techniques such as inkjet printing ${ }^{5,6}$ or spray coating, ${ }^{7,8}$ such inks can be used to prepare a range of films, coatings or patterned structures. In particular, applications in areas such as printed electronics will require the production of conductive films or traces. Here, defect free graphene performs particularly well, giving high conductivity

${ }^{a}$ Centre for Research on Adaptive Nanostructures and Nanodevices (CRANN), Trinity College Dublin, Dublin 2, Ireland

${ }^{b}$ Thomas Swan \& Co. Ltd., Rotary Way, Consett, County Durham, DH8 7ND, UK ${ }^{c}$ School of Physics, Trinity College Dublin, Dublin 2, Ireland.E-mail: colemaj@tcd.ie $\dagger$ Electronic supplementary information (ESI) available. See DOI: $10.1039 / \mathrm{c} 4 \mathrm{nr} 03560 \mathrm{~g}$ structures without high temperature post treatments. ${ }^{5}$ Thus, it is clear that large scale production techniques for defect-free graphene are urgently required.

Defect free graphene is generally produced by sonicating graphite powder either in certain solvents ${ }^{9-16}$ or aqueous surfactant ${ }^{17-23}$ solutions. The sonication tends to break up the graphite crystallites as well as exfoliating them to give large number of graphene nanosheets. ${ }^{11}$ Raman spectroscopy ${ }^{15,24,25}$ shows this method to produce negligible quantities of basal plane defects while XPS shows the flakes to be un-oxidised. ${ }^{14}$ While the graphene produced by this method is of high quality, some major problems remain. Most important is the fact that processes involving sonication have limited scalability. The concentration, $C$, of sonication-exfoliated graphene tends to scale roughly linearly with the inputted energy density: $C \propto(E /$ $V)=P t / V$, where $E, V, P$ and $t$ are the total inputted energy, the liquid volume, the input power and the sonication time respectively. ${ }^{23,26}$ This means the production rate $\left(P_{\mathrm{R}}=V C / t\right)$ is roughly independent of volume. This is a significant problem as it means the simplest strategy for scaling up a process, increasing the production rate by increasing the volume, is unavailable. This makes it unlikely that sonication exfoliation of graphene will ever be scaled up industrial levels. This means that alternative methods of providing an energy supply to drive the exfoliation of graphene in liquids are urgently required.

Recently, it was shown that graphene could be exfoliated in a liquid under shear. ${ }^{27}$ Subsequently, we have demonstrated a method of shear-exfoliation of graphite to give graphene that is industrially scalable. This involves using a high-shear, rotorstator mixer to produce graphene in stabilising solvents as well 
as polymer- or surfactant solutions. ${ }^{26}$ The importance of this method is that the production rate scales as $P_{\mathrm{R}} \propto V^{1.6}$ which means scale-up can be achieved simply by increasing the liquid volume. However, there are certain problems with this approach. Worryingly, this method gives relatively low concentrations $\left(C \leq 0.1 \mathrm{mg} \mathrm{ml}^{-1}\right.$ ), limiting the production rate. Most importantly, the concentration scales with mixing time as $C \propto$ $t^{n}$ where $n<1$. As a result, the production rate falls with mixing time, limiting the overall output.

However, we believe the inherent advantages of shear exfoliation far outweigh these difficulties. It is likely that the problems outlined above are related to the details of the interaction of the liquid with the rotor-stator. It is possible that other methods exist to generate the high shear in a scalable manner without the problems described above. Measurements both in our group ${ }^{26}$ and by others ${ }^{28}$ have shown that graphite can be exfoliated to give graphene using a very simple, high speed rotating blades. Here we study this process in greater detail using a kitchen blender to produce shear. We find a linear increase in concentration with time leading to concentrations which are ten times higher than those achieved with the high shear, rotor-stator mixer. In addition, the concentration falls very slowly with volume, leading to a production rate that increases with increasing volume. Comparison to the literature shows this process to be superior to sonication as a method for producing graphene.

\section{Results and discussion}

\section{Blender exfoliation of graphene}

To facilitate ease of production and minimise cost, the equipment used for graphene production should be as simple as possible. To demonstrate this, we wanted to use the most basic form of shear mixer possible. As such we purchased a Kenwood BL370 series kitchen blender in a local high street shop (Fig. 1A). This consists of a tapered jug, $\sim 100 \mathrm{~mm}$ diameter at the base, expanding to $\sim 125 \mathrm{~mm}$ at the top with a capacity of 1.6 $\mathrm{L}$ (though working volume is only $1.2 \mathrm{~L}$ ). It is fitted with four baffles running the full height of the jug, fixed to the walls projecting $4 \mathrm{~mm}$ into the liquid and each with a thickness of 1 $\mathrm{mm}$. The function of the baffles is to suppress rotation of the liquid as a whole as well as to increase turbulence. The impeller consists of 4 blades. Two are angled slightly above horizontal, with a diameter of $53 \mathrm{~mm}$, while two are angled below horizontal with a diameter of $58 \mathrm{~mm}$ (Fig. 1B). It is fitted with a 400 W motor, with a nominal rotational speed of $21 \pm 3 \mathrm{krpm}$. However, we measured the actual rate to be $18 \mathrm{krpm}$ (see SI $\dagger$ ). Because the blender is predominately made from plastic, we decided to attempt to exfoliate graphite in an aqueous surfactant solution. Aiming to keep the process as un-sophisticated as possible we chose the household surfactant, Fairy washing-up liquid (FL), as our dispersant.

We performed initial trials by adding water $(V=500 \mathrm{ml})$, graphite (concentration: $\left.C_{\mathrm{i}}=20-100 \mathrm{mg} \mathrm{ml}^{-1}\right)$ and $\mathrm{FL}\left(C_{\mathrm{i}} / C_{\mathrm{FL}}=\right.$ 1-100) to the blender and blending for times in the range $t=5-$ $30 \mathrm{~min}$. When the blender is turned on, the graphite is rapidly lifted off the bottom of the vessel. The liquid turns a black/gray
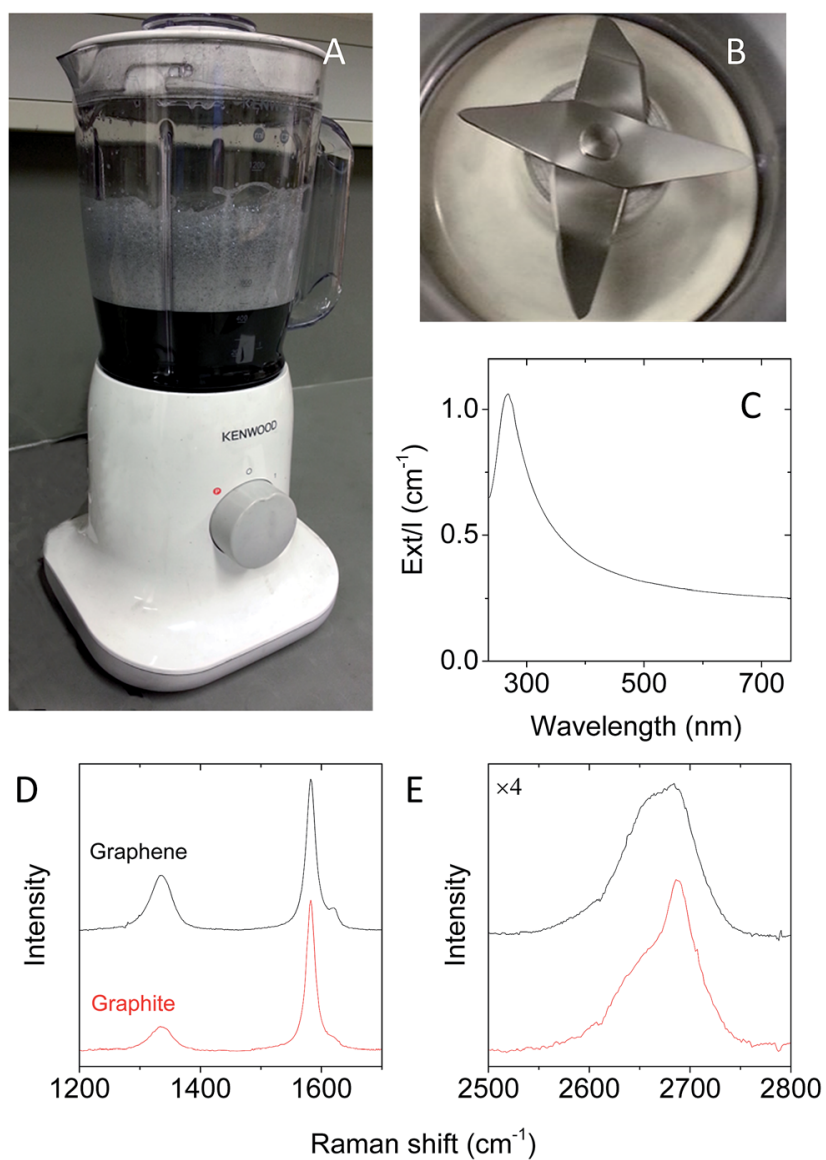

Fig. 1 (A) The Kenwood BL370 series kitchen blender used in this work photographed during a mix. The blender is mixing graphite powder in an aqueous surfactant solution. The surfactant is the household detergent, Fairy Liquid. Note the presence of significant amounts of foam. (B) A photograph of the rotating blade supplied with this blender. (C) Typical extinction spectrum measured for a dispersion after centrifugation. (D-E) Raman spectrum of the graphene produced in this work with that of the graphite shown for comparison ( $\lambda_{\text {ex }}=633$ $\mathrm{nm})$. Panel (D) shows the D- $\left(\sim 1340 \mathrm{~cm}^{-1}\right)$ and $\mathrm{G}$-bands $\left(\sim 1680 \mathrm{~cm}^{-1}\right)$ while panel (E) shows the 2D-bands $\left(\sim 2670 \mathrm{~cm}^{-1}\right)$. The 2D-band intensities have been multiplied by a factor of 4 for clarity. The data in (C-E) was recorded for a dispersion prepared using $C_{\mathrm{i}}=20 \mathrm{mg} \mathrm{ml}^{-1}$, $C_{\mathrm{FL}}=2.5 \mathrm{mg} \mathrm{ml}^{-1}, \mathrm{~V}=500 \mathrm{ml}, t=60 \mathrm{~min}, N=18 \mathrm{krpm}$.

colour as the graphite starts to disperse and small bubbles begin to form (Fig. 1A). In general, a considerable amount of foam is produced which can overflow the vessel. In some cases a vortex, which is typically a few centimetres deep, is observed although in the majority of cases, the foam completely obscures it. After mixing, the liquid slowly turns black as the bubbles dissipate. To investigate the nature of the black liquid produced in this way, it was centrifuged at $1500 \mathrm{rpm}$ for 45 minutes and the supernatant collected.

As an initial test, we measured the optical extinction spectrum of each dispersion with a typical example shown in Fig. 1C, normalised to cell length, $l$. (N.B. the extinction, Ext, is defined by the transmittance, $T$, where $T=10^{- \text {Ext }}$. We refer to this quantity as extinction as, for nanosheet dispersions, it contains contributions from both absorbance and scattering ${ }^{29}$ ). 
This spectrum is typical of graphene dispersions, displaying a peak at $\sim 265 \mathrm{~nm}$ but flattening at higher wavelengths. ${ }^{30}$ Below, we will use such spectra to calculate the graphene concentration on a range of dispersions.

We confirm that the dispersed material is graphene by performing Raman spectroscopy on films of dried, deposited graphene. A typical Raman spectrum is shown in Fig. 1D and $E$ with the graphite spectrum shown for comparison $\left(\lambda_{\mathrm{ex}}=633 \mathrm{~nm}\right)$. As is typical for solution-processed graphene, ${ }^{\mathbf{1 4 , 1 5}}$ this spectrum has three main peaks: the D-band $\left(\sim 1330 \mathrm{~cm}^{-1}\right)$, the G-band $\left(\sim 1560 \mathrm{~cm}^{-1}\right)$ and the 2D-band $\left(\sim 2650 \mathrm{~cm}^{-1}\right)$. The $2 \mathrm{D}$ band is reasonably symmetric but less intense than the $\mathrm{G}$ band, indicating the samples to be dominated by few-layer graphene. ${ }^{31}$ The D-band is generally associated with defects ${ }^{31}$ and was found here to be narrow (FWHM $\sim 45 \mathrm{~cm}^{-1}$ ) and considerably less intense than the $\mathrm{G}$ band. The $\mathrm{D}: \mathrm{G}$ intensity ratio was always in the range $0.3-0.7$. This is similar to what is usually found for graphene flakes with lateral size hundreds of $\mathrm{nm}$ and is consistent with the defects being associated with the nanosheet edges rather than basal plane defects. ${ }^{15,24-26}$ This is an important point as it suggests shear exfoliation using the rotating-blade mixer does not introduce basal plane defects. This is consistent with the detailed study using a high-shear rotor-stator mixer published previously which clearly demonstrated that exfoliation of graphene by shear does not introduce basal plane defects. ${ }^{26}$

To further characterise the flakes, we performed microscopic characterisation. Shown in Fig. 2A and B are TEM images which clearly show thin nanosheets of few-layer graphene. Over the course of this work, we imaged in excess of 400 nanosheets. All of these nanosheets appear very similar to few-layer graphene prepared by sonication of graphite in solvents ${ }^{9-16}$ or aqueous surfactant ${ }^{17-23}$ solutions. However, we have observed one clear difference between the nanosheets analysed in this study and those prepared by sonication: while sonication-exfoliated graphene nanosheets are sometimes folded, such folding is much more common in graphene prepared with the blender. In fact from a subset of 250 flakes examined by TEM, 54\% were clearly folded. It is likely that the larger fraction of folded flakes in mixer-exfoliated graphene relative to sonication exfoliated graphene reflects differences in the fluid dynamics of the two systems. We also performed AFM analysis on a small number of dispersions. Typical images are shown in Fig. 2C and D. From these images it is clear that the flakes are relatively thin (see below).

From the TEM images, we measured the longest dimension (flake length, $L$ ) for 50 flakes each in samples produced using a range of combinations of processing parameters $\left(C_{\mathrm{i}}, C_{\mathrm{i}} / C_{\mathrm{FL}}, t\right)$. Shown in Fig. $2 \mathrm{E}$ is a typical flake length histogram $\left(C_{\mathrm{i}}=20 \mathrm{mg}\right.$ $\mathrm{ml}^{-1}, C_{\mathrm{FL}}=2.5 \mathrm{mg} \mathrm{ml}^{-1}, V=500 \mathrm{ml}, t=60 \mathrm{~min}, N=18 \mathrm{krpm}$, details see Methods) showing a mean length of $630 \mathrm{~nm}$, reasonably similar to that found for sonication-exfoliated graphene (depending on processing parameters). All combinations of processing parameters gave similar flake size distributions, with some variation in the mean. Over the entire study, maximum flake length observed was $\sim 3.3 \mu \mathrm{m}$. A future study will investigate in more detail the relationship between flake size and processing parameters.
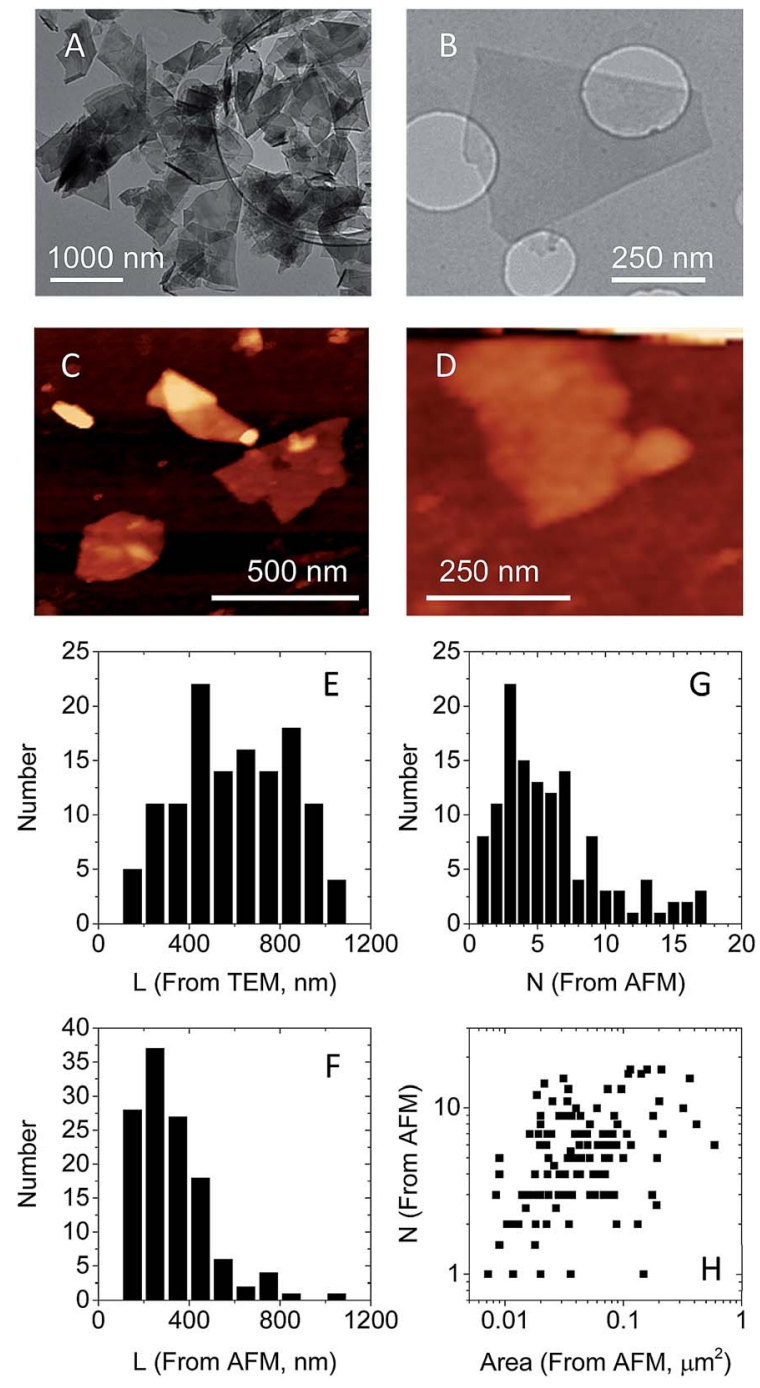

Fig. 2 Microscopic analysis of nanosheets found in a typical mixerexfoliated dispersion $\left(C_{\mathrm{i}}=20 \mathrm{mg} \mathrm{ml}^{-1}, C_{\mathrm{FL}}=2.5 \mathrm{mg} \mathrm{ml}^{-1}, \mathrm{~V}=500 \mathrm{ml}\right.$, $t=60 \mathrm{~min}, N=18 \mathrm{krpm}$ ). (A) Wide-field TEM image of graphene nanosheets produced by the mixer. (B) An individual, very thin flake. (C) AFM image of graphene nanosheets. The height of the right-most flake is consistent with bi-layer graphene. (D) AFM image of a nanosheet whose height is consistent with monolayer graphene. (E-F) Length distributions of nanosheets as measured by (E) TEM and (F) AFM. (G) Flake thickness distribution, represented as number of monolayers per flake, $N$, as measured by AFM. (H) Flake thickness plotted versus nanosheet area (both measured by AFM).

We have also performed statistical length and thickness analysis for the same sample as presented in Fig. $2 \mathrm{E}\left(C_{\mathrm{i}}=20 \mathrm{mg}\right.$ $\mathrm{ml}^{-1}, C_{\mathrm{FL}}=2.5 \mathrm{mg} \mathrm{ml}^{-1}, V=500 \mathrm{ml}, t=60 \mathrm{~min}, N=18 \mathrm{krpm}$, details see Methods) by AFM (Fig. 2F-H). Interestingly, the AFM length data shows flakes which are smaller than suggested by TEM (mean length of $320 \mathrm{~nm}$ ). We believe the discrepancy arises from the fact that TEM length statistics on polydisperse samples often underrepresent small flakes, as these can be difficult to observe/image and count fairly, especially when a dispersion also contains significantly larger nanosheets. Incidentally, this highlights the problem of using microscopy to perform accurate measurements of sizes for objects which have 
broad size distributions. In addition, one must consider whether number average or weight averages are more appropriate for such broad distributions. The weight average flake length, extracted from the AFM data (calculated using $\left\langle L^{3} N\right\rangle$ / $\left\langle L^{2} N\right\rangle$ ), was $\sim 520 \mathrm{~nm}$, much closer to the TEM number average. This suggests the discrepancy between TEM and AFM data is due to the relative absence of small flakes in the TEM statistics.

The AFM data can be used to measure the flake height. ${ }^{26,29}$ Shown in Fig. 2G is a histogram of flake heights (represented by number of monolayers per flake, $N)$ as measured for the $\left(C_{\mathrm{i}}=\right.$ $20 \mathrm{mg} \mathrm{ml}^{-1}, C_{\mathrm{FL}}=2.5 \mathrm{mg} \mathrm{ml}^{-1}, V=500 \mathrm{ml}, t=60 \mathrm{~min}, N=18$ $\mathrm{krpm})$ sample. This data shows the bulk of the flakes to have 1 $\leq N \leq 10$ with a mean of $\langle N\rangle=6$, very close to that reported by Paton et al. for shear exfoliated graphene. ${ }^{26}$ We observed only a small number of monolayers $(\sim 6 \%)$ with an example shown in Fig. 2D. Previously, it has been demonstrated that for sonication-exfoliated nanosheets, the thickness appears to scale with nanosheet area. ${ }^{29,32}$ This is unfortunate as it makes it difficult to produce large, yet thin, nanosheets. Shown in Fig. $2 \mathrm{H}$ is a plot of flake thickness, $N$, versus flake area (approximated by $L^{2}$ ) measured for the $\left(C_{\mathrm{i}}=20 \mathrm{mg} \mathrm{ml}^{-1}, C_{\mathrm{FL}}=2.5 \mathrm{mg} \mathrm{ml}^{-1}, V=\right.$ $500 \mathrm{ml}, t=60 \mathrm{~min}, N=18 \mathrm{krpm}$ ) sample by AFM. Here the relationship between $N$ and area is much weaker than previously reported, suggesting shear exfoliation might be used to produce large, thin nanosheets.

\section{Graphene concentration and production rate}

The main advantage of using a rotating-blade mixer to produce graphene is that it has the potential to process relatively large liquid volumes, reasonably quickly. In contrast, sonic tips can be used to effectively process volumes no larger than a few $100 \mathrm{ml}$ leading to low production rates. ${ }^{21,33}$ While sonic baths can be used to process hundreds of millilitres, the power transfer from bath to liquid is relatively poor, leading to long exfoliation times and so low production rates. ${ }^{\mathbf{1 4 , 1 5 , 2 0}}$ Using the Kenwood mixer, volumes of up to $1.2 \mathrm{~L}$ can be processed effectively, as the rotating blade transfers mechanical energy directly into the liquid..$^{34,35}$ Thus, it is important to explore quantitatively how much graphene can be produced using such a mixer.

To do this, we investigated how the concentration of graphene produced in the mixer varies with processing parameters such as $C_{\mathrm{i}}$ and $t$ as well as blade rotation rate, $N$, and liquid volume, $V$. However, first it is necessary to identify the optimum surfactant content. It has been observed that the concentration of exfoliated graphene displays a peak at an optimum surfactant content. ${ }^{19,20}$ To test this, we mixed graphite at two initial concentrations $\left(C_{\mathrm{i}}=20\right.$ and $\left.100 \mathrm{mg} \mathrm{ml}^{-1}\right)$ in $V=500 \mathrm{ml}$ of aqueous surfactant solution for $t=30$ minutes with $N=18$ $\mathrm{krpm}$. For each $C_{\mathrm{i}}$, this was performed for a range of surfactant concentrations, $C_{\mathrm{FL}}$. After mixing, the resultant dispersions were centrifuged and the supernatant concentration measured by extinction spectroscopy, obtaining spectra such as that in Fig. 1C. The measured concentration, $C$, has been plotted versus $C_{\mathrm{FL}}$ in Fig. 3A for both values of $C_{\mathrm{i}}$. In each case the dispersed concentration showed a clear peak which appeared at higher $C_{\mathrm{FL}}$ for the samples with higher $C_{\mathrm{i}}$. This suggests that the
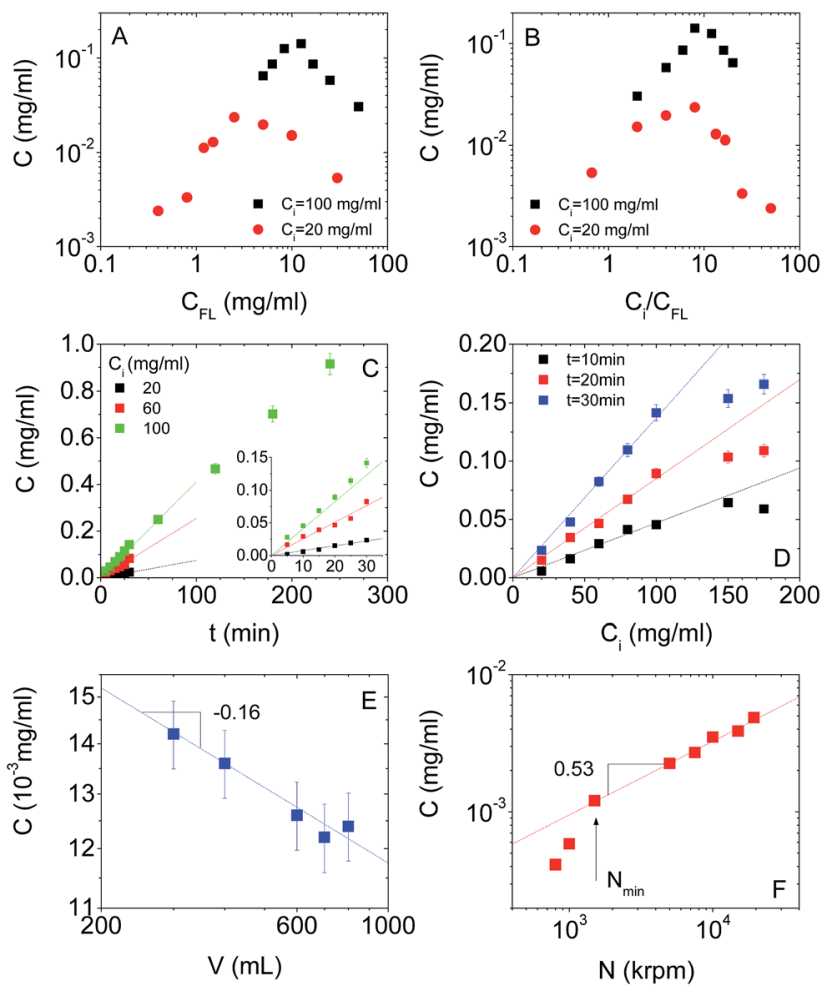

Fig. 3 Concentration of mixer dispersed graphene plotted versus (A) surfactant concentration, $C_{\mathrm{FL}},(\mathrm{B})$ ratio of graphite to surfactant concentration, $C_{\mathrm{i}} / C_{\mathrm{FL}}$, (C) mixing time, $t$, (D) graphite concentration, $C_{\mathrm{i}}$, (E) liquid volume, $V$, and (F) blade speed, $N$. The inset in (C) shows times up to $35 \mathrm{~min}$. The fit lines in (E) and (F) are power laws while those in (C) and (D) are linear. In all cases the uncertainty in concentration measurements was $\sim 5 \%$. In most cases, the error bars are smaller than the symbol. N.B. the vertical axis in (E) is actually plotted logarithmically although it appears linear due to the small range.

optimum surfactant concentration depends on $C_{\mathrm{i}}$ and implies that it may be more important to optimise the ratio $C_{\mathrm{i}} / C_{\mathrm{FL}}$. To test this, we have plotted $C$ versus $C_{\mathrm{i}} / C_{\mathrm{FL}}$ in Fig. 3B. In this case we see that both data sets show a peak at the same value of $C_{\mathrm{i}} /$ $C_{\mathrm{FL}}=8$. For the rest of this study, we use this optimised ratio.

Once the $C_{\mathrm{i}} / C_{\mathrm{FL}}$ is fixed, the parameters which might be expected to control the graphene concentration are the mixing time, $t$, the graphite concentration, $C_{\mathrm{i}}$, the blade rotation rate, $N$, and the liquid volume, $V$. Initially, we focused on the effects of $t$ and $C_{\mathrm{i}}$, as these parameters can easily be controlled over a relatively broad range. Keeping $N=18 \mathrm{krpm}, V=500 \mathrm{ml}$ and $C_{\mathrm{i}} /$ $C_{\mathrm{FL}}=8$, we prepared a set of dispersions by mixing for $t=5 \mathrm{~min}$ at a range of graphite concentrations in the range $20 \leq C_{\mathrm{i}} \leq 175$ $\mathrm{mg} \mathrm{ml} \mathrm{m}^{-1}$. After mixing, the dispersions were centrifuged, the supernatant collected and the graphene concentration measured. This whole procedure was then repeated for mixing times of $t=10,15,20,25$ and 30 minutes. In general we were limited to relatively short mixing times because of technical issues associated with the blender (see Methods). However for $C_{\mathrm{i}}=100 \mathrm{mg} \mathrm{ml}^{-1}$, we also measured $C$ for $t=60,120,180$ and 240 minutes. Shown in Fig. 3C are plots of $C$ versus $t$ for a subset of $C_{\mathrm{i}}$ values. It is clear that graphene concentration increases linearly with mixing time for all data sets studied. This is in 
contrast to a number of papers which have described sonication-induced exfoliation and have reported a sublinear increase in concentration with sonication time, consistent with $C \propto \sqrt{t}$, for both solvent and surfactant stabilised graphene..$^{15,20,25,33}$ However, for high shear mixing, which is perhaps more relevant here, the picture is more complicated. When graphene is produced using a high shear mixer, the concentration always scales as $C \propto t^{n} .{ }^{26}$ When mixing in solvents such as NMP, $n \approx$ 0.6 , similar to what is found for sonication. However, for shear exfoliation of graphene in surfactant solutions, $n$ is higher, approaching $1,{ }^{26}$ as we find here. Thus, it is not clear if the linear behaviour observed here is due to the specific details of the exfoliation process occurring in the blender or if it is more general to shear exfoliation in the presence of surfactants. The highest concentration observed was $\sim 1 \mathrm{mg} \mathrm{ml}^{-1}$, much higher than the maximum value of $\sim 0.1 \mathrm{mg} \mathrm{ml}^{-1}$ found using the rotor-stator mixer. ${ }^{26}$ This is also considerably higher than the maximum graphene concentration observed in the earliest work on sonication exfoliation of graphene, ${ }^{\mathbf{1 4}}$ and in line with concentrations achievable today using sonication. ${ }^{15}$ We note that even at this relatively high graphene concentration, the dispersion contains $\sim 12$ times more surfactant than graphene. However, experiments show that the vast majority of the surfactant can be removed by washing a vacuum-filtered film (see $\mathrm{SI}^{\dagger}$ ) with the washed film containing $\sim 20$ to $30 \%$ surfactant, equivalent to $98 \%$ of the surfactant being removed.

Shown in Fig. 3D is a graph of $C$ versus $C_{\mathrm{i}}$ for a subset of $t$ values $\left(N=18 \mathrm{krpm}, V=500 \mathrm{ml}\right.$ and $\left.C_{\mathrm{i}} / C_{\mathrm{FL}}=8\right)$. It is clear that graphene concentration increases linearly with graphite concentration for all data sets studied. However, for all mixing times, the concentration begins to saturate for $C_{\mathrm{i}}>$ $100 \mathrm{mg} \mathrm{ml}^{-1}$.

We also tested the effect of liquid volume by blending while the jug contained different liquid volumes from 300-800 $\mathrm{ml}\left(C_{\mathrm{i}}\right.$ $=20 \mathrm{mg} \mathrm{ml}{ }^{-1}, t=15 \mathrm{~min}, N=18 \mathrm{krpm}$ and $\left.C_{\mathrm{i}} / C_{\mathrm{FL}}=8\right)$. Plotted in Fig. $3 \mathrm{E}$ is a graph of $C$ versus $V$ on a $\log -\log$ scale showing a slight fall-off in $C$ as the liquid volume is increased. The concentration falls off roughly as a power law as observed for graphene prepared in a high shear mixer. ${ }^{26}$ However, here the exponent is -0.16 , much smaller that the values of -0.47 and -0.68 found for high shear mixing of graphene stabilised in aqueous surfactant solutions and solvents respectively. This small exponent is of interest as it means the volume can be increased without large decreases in the concentration of graphene produced, an important consideration when it comes to large scale production. In this regard, this blender is superior to high shear mixers. In addition, this small exponent implies that exfoliation occurs throughout much of the jug volume rather than just in the vicinity of the blade.

Exploring the effect of blade rotation rate is more challenging because the blade is designed to work at a fixed rate $(N$ $=18 \mathrm{krpm}$ ). However, we were able to control $N$ from $0.8 \mathrm{krpm}$ to $18 \mathrm{krpm}$ (see $\mathrm{SI} \dagger$ ). We prepared a number of samples with varying $N$ and measured $C\left(C_{\mathrm{i}}=20 \mathrm{mg} \mathrm{ml}^{-1}, t=5 \mathrm{~min}, V=300\right.$ $\mathrm{ml}$ and $\left.C_{\mathrm{i}} / C_{\mathrm{FL}}=8\right)$. Shown in Fig. $3 \mathrm{~F}$ is a graph of $C$ versus $N$ on a $\log$ - $\log$ scale. For values of $N>N_{\min } \sim 1800 \mathrm{rpm}$, this data follows a power law consistent with $C \propto N^{0.53}$. However, for $N<$
$N_{\text {min }}$, the concentration falls off. Interestingly, this is similar to what was observed for graphene produced by high shear mixing in solvents where $C \propto N^{1.13}$ above a critical value of $\sim 10^{4} \mathrm{rpm}^{26}$ However, when high shear mixing was performed using the rotor-stator in aqueous surfactant solutions, $C \propto N^{2.52}$ was observed. ${ }^{26}$

While the exponent observed for high-shear mixing in surfactants is not understood, the exponent for high-shear mixing in solvents has been explained as a reflection of the empirical observation that the graphene yield scales roughly as the square root of inputted energy density. ${ }^{26}$ The power input of a rotating-blade mixer is given by ${ }^{35}$

$$
P=N_{\mathrm{p}} \rho N^{3} D^{5}
$$

where $N_{\mathrm{p}}$ is a dimensionless constant which is known to have values of $\sim 0.1$ to 0.3 for blades similar to that used here (i.e. the EkatoMizer and the LE-20 described in ref. 36). On a per unit liquid mass basis, $P / M$, is given by

$$
\frac{P}{M}=\frac{N_{\mathrm{p}} N^{3} D^{5}}{V}
$$

Interestingly, for the blender, the measured exponents relating concentration to $N$ and $V$ have a ratio $0.53 /(-0.16) \approx$ -3.3 , very close to that found in eqn (2). This shows our data to be roughly consistent with $C \propto(P / M)^{1 / 6}$. However, diameter dependent data showing $C \propto D^{5 / 6}$ would be required to demonstrate this more convincingly. Unfortunately, using the kitchen blender described above, we have no scope to vary $D$.

The data described in Fig. 3 can be used to calculate parameters that are directly relevant to graphene production. We can define the yield as the ratio of concentration of few layer graphene to initial graphite concentration, $Y=C / C_{\mathrm{i}}$. This can be found from the slope of the linear regions in Fig. 3D and is plotted versus mixing time in Fig. 4A (closed symbols). This data shows excellent linearity, reaching $Y=0.14 \%$ after 30 minutes. To test if the yield can be increased further, we performed one very long mix $\left(t=4 \mathrm{~h}, V=500 \mathrm{ml}, C_{\mathrm{i}}=100 \mathrm{mg}\right.$ $\left.\mathrm{ml}^{-1}, N=18 \mathrm{krpm}, C_{\mathrm{i}} / C_{\mathrm{FL}}=8\right)$, removing aliquots after each hour. These were centrifuged, the concentration measured and the yield determined. The yield for these samples has been plotted on Fig. 4A, alongside the data described above. This data follows the same linear trend as before, approaching $Y=$ $1 \%$ after 4 hours mixing. While this yield is relatively low compared to that achievable with sonication (yields of $5 \%$ have been observed when bath sonicating for $3 \mathrm{~h}$, increasing to $45 \%$ after $\sim 200 \mathrm{~h}),{ }^{15}$ it is much higher than the maximum yield achieved by high-shear mixing $(\sim 0.2 \%) .{ }^{26}$ It is worth noting that such low yields are not as problematic as they appear, as unexfoliated graphite can be recycled and used as new starting material. ${ }^{\mathbf{1 4 2 6}}$ While it would be interesting to see how far this linear trend extends, technical limitations of the mixer make long mixes challenging (see Methods). However, it is clear that rotating blade mixers are capable of attaining impressive yields (see below for detailed comparison to other production methods). 

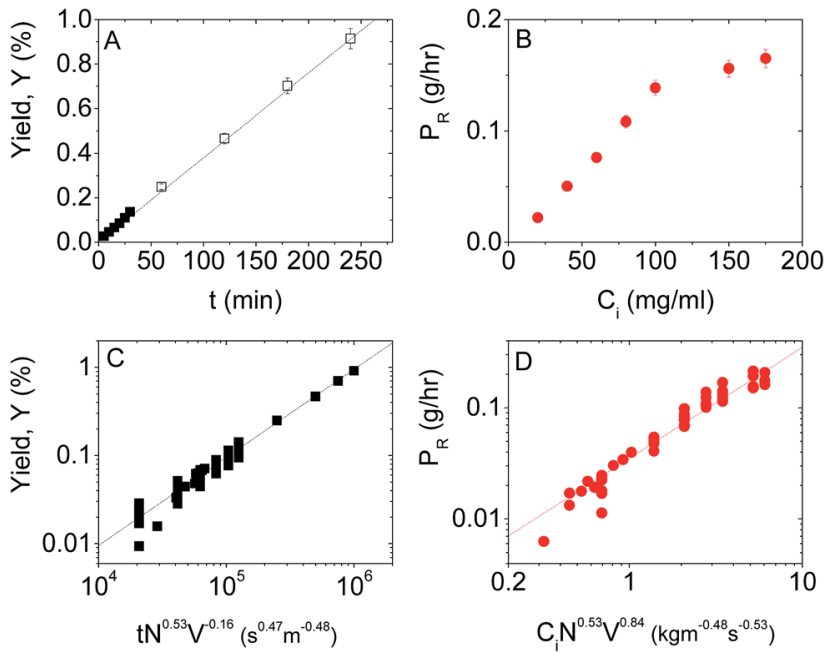

Fig. 4 (A) Graphene yield $\left(Y=C / C_{i}\right)$ plotted versus mixing time. The solid data points are calculated from the slopes of $C$ versus $C_{i}$ curves such as those in Fig. 3D. The open symbols come from individual concentration measurements $\left(V=500 \mathrm{ml}, C_{i}=100 \mathrm{mg} \mathrm{ml}^{-1}, N=18\right.$ krpm, $\left.C_{\mathrm{i}} / C_{\mathrm{FL}}=8\right)$. (B) Production rate $\left(P_{\mathrm{R}}=V C / t\right)$ plotted versus graphite concentration. (C) Yield and (D) production rate master curves plotted versus the scaling factors discussed in the text.

Another important parameter is the graphene production rate which is given by $P_{\mathrm{R}}=V C / t$ and represents the total mass of few-layer graphene produced per unit time. The production rate can be found from $C$ versus $t$ plots such as those in Fig. 3C. Because of the linear relationship between $C$ and $t$, the production rate is constant over time. This is a considerable advantage over exfoliation in solvents using high-shear mixers where $P_{\mathrm{R}}$ falls off with mixing time (because $C \propto t^{n}$ with $n<$ 1). ${ }^{26}$ We plot the production rate versus $C_{\mathrm{i}}$ in Fig. $4 \mathrm{~B}$. As was suggested by the data in Fig. $3 \mathrm{D}, P_{\mathrm{R}}$ increases linearly with initial graphite concentration up to $C_{\mathrm{i}}=100 \mathrm{mg} \mathrm{ml}^{-1}$ after which it falls off somewhat. We observed a maximum production rate of $\sim 0.15 \mathrm{~g} \mathrm{~h}^{-1}$, comparable to that achieved in lab trials with a high shear mixer $\left(0.001-1 \mathrm{~g} \mathrm{~h}^{-1}\right)$ and significantly higher than that typically achieved by sonication (see below and ref. 26).

The data in Fig. 3 shows the concentration of graphene produced in the blender to be proportional to $t, C_{\mathrm{i}}$ and $N^{0.53}$ and scale with $V^{-0.16}$. This allows us to write $C \propto C_{\mathrm{i}} t N^{0.53} V^{-0.16}$. Using this expression, we can write expressions for $Y$ and $P_{\mathrm{R}}$ in terms of scaling factors: $Y \propto t N^{0.53} V^{-0.16}$ and $P_{\mathrm{R}} \propto C_{\mathrm{i}} N^{0.53} V^{0.84}$. This volume dependence is particularly important as it means the production rate can be increased by scaling up the volume. We can test the validity of these expressions by plotting $Y$ and $P_{\mathrm{R}}$ versus the appropriate scaling factor for all data sets in Fig. 3C and D. In each case, we find all data to collapse onto a master curve with good linearity, as indicated by the dashed lines. This allows us to predict how $C, Y$ and $P_{\mathrm{R}}$ vary over almost the entire parameter space. However, it is worth remembering that these parameters should also scale with the rotor diameter, ${ }^{26,34,35}$ a parameter which is of course fixed in this study.

\section{Comparison with sonication}

It is important to benchmark the performance of this mixer against the standard technique for producing defect-free graphene in liquids i.e. sonication of graphite in solvents or surfactant solutions. A number of papers have described how the graphene concentration increases with sonication time. However, concentration is not a good metric for graphene production because high concentrations may only be attainable in low volumes resulting in small quantities of graphene produced. For a given sonication time, sonic tips can achieve high concentrations in low volumes while baths can achieve low concentrations in higher volumes so neither is clearly superior in terms of mass produced. A better metric is the mass of graphene produced: $M=C V$. We therefore compare data for mass of graphene produced as a function of time using the mixer $\left(C_{\mathrm{i}}\right.$ $\left.=100 \mathrm{mg} \mathrm{ml}^{-1}, V=500 \mathrm{ml}, N=18 \mathrm{krpm}, C_{\mathrm{i}} / C_{\mathrm{FL}}=8\right)$ to data taken from the literature for graphene produced by sonication of graphite in the solvent NMP using both tip ${ }^{33}$ and bath ${ }^{15}$ and graphene prepared by bath-sonication of graphite in an aqueous solution of the surfactant; sodium cholate. ${ }^{20} \mathrm{We}$ also produced graphene by sonication in an aqueous FL solution for a direct comparison (see Methods). This data is shown in Fig. 5 and makes it clear that the blender used here is superior to both tip- and bath-sonication in either solvent or surfactant environments. A number of points are of interest. Because the mass produced by the blender increases linearly with time while the mass produced by sonication generally scales sub-linearly with time, the relative superiority of the blender increases with mixing time. Also, blender-exfoliation in surfactant is superior to sonication in solvents, even though sonication in solvents is far superior to sonication in aqueous surfactant solutions.

It is worth emphasising that these results, impressive though they are, were achieved using a Kenwood kitchen blender which cost $€ 40$. In contrast, sonic baths cost hundreds of Euro while sonic tips cost thousands of Euro. At its most basic level, the

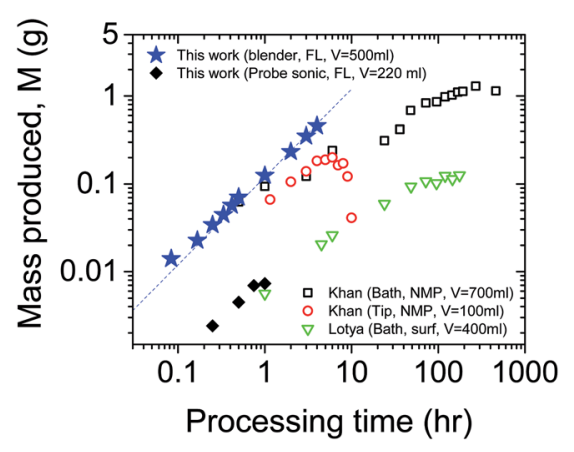

Fig. 5 Comparison to other work describing the preparation of graphene of similar quality. The mass was found from $m=C V$ where $C$ is the concentration and $V$ is the liquid volume. The legend gives the first author of the paper ${ }^{15,20,33}$ from which the data was taken as well as the energy source (sonic tip versus sonic bath), the stabilising liquid (the solvent $\mathrm{N}$-methyl-pyrrolidone or an aqueous surfactant solution) and the liquid volume used. Also shown is data for production of graphene by probe-sonication of graphite in an aqueous FL solution (black diamonds) 
blender used here is just a combination of a rotating blade and a vessel to contain the liquid. A number of companies supply industrial versions of rotating blade mixers. These have the advantage that they can be used with a range of containers. For example, metal containers could be used allowing the production of graphene in solvents such as NMP. Under such circumstances, it is likely that the production rate could be increased far beyond what is described here.

\section{Exfoliation mechanism}

It is important to consider the exfoliation mechanism. By studying shear exfoliation of graphite to give graphene in both laminar and turbulent flow, ${ }^{26}$ Paton et al. have shown that graphene nanosheets of the size produced here are only produced once the local shear rate is greater than a critical value $\dot{\gamma}_{\mathrm{c}} \sim 10^{4} \mathrm{~s}^{-1}$. In a high-shear rotor-stator mixer, very high shear rates are achieved in the gap between the rotor and stator and in the holes in the stator. ${ }^{26,37}$ This means graphene production is probably localised in the vicinity of the rotor-stator. However, for a rotating-blade mixer no such well-defined, highly localised regions of high shear exist. Although the average shear rate probably falls with distance from the blade, the high shear zone is not as well-defined as it is in a rotor-stator mixer.

However, in a rotating blade mixer, it is likely that turbulence is responsible for the presence of high shear rates which are not localised in any single part of the vessel. The Reynolds number (Re) associated with the rotating blade ${ }^{35}$ is $\operatorname{Re}_{\text {Blade }}=N D^{2} \rho / \eta$, where $\rho$ and $\eta$ are the liquid density $\left(1000 \mathrm{~kg} \mathrm{~m}^{-3}\right)$ and viscosity (0.001 Pa s) respectively. When $N$ is at its maximum value $(18 \mathrm{krpm})$, then $\operatorname{Re}_{\text {Blade }}=10^{6}$, far above the value of $\operatorname{Re}_{\text {Blade }} \sim$ $10^{4}$ where turbulence becomes fully developed. ${ }^{37}$ We suggest that it is this turbulence that produces local shear rates that are high enough to result in exfoliation.

In a turbulent system the energy inputted at the macro-scale (here by the rotating blade) is transferred from large to small length scales (the so-called inertial subrange) in an almost dissipation-less cascade through eddies of various sizes. ${ }^{\mathbf{3 8 - 4 0}}$ The smallest eddies exist at a length-scale known as the Kolmogorov length, $\lambda$, below which viscous effects dominate over inertial ones (the so-called viscous sub-range). The inputted energy is eventually dissipated as heat at length scales below $\lambda .^{41}$ The Kolmogorov length is given by $\lambda=\left(\eta^{3} \rho^{-3} \varepsilon^{-1}\right)^{1 / 4}$ where $\varepsilon$ is the mean rate of dissipation of turbulent energy per unit mass. ${ }^{\mathbf{4 1}}$ Making the approximation that $\varepsilon$ is equal to the input power per unit mass, $\varepsilon \approx P / M=P / \rho V$ (for this blender $P=400 \mathrm{~W}$ and, for most mixes performed, $V=500 \mathrm{ml}$ ), then $\lambda=6 \mu \mathrm{m}$. Because the graphene nanosheets produced in the blender are much smaller than this, it is likely that exfoliation is associated with processes occurring in the viscous subrange (i.e. length scales below $\lambda$ ).

In the viscous subrange, turbulent motion results in strong spatial and temporal variation of the local shear rate. ${ }^{\mathbf{4 1 , 4 2}}$ However, the mean turbulent shear rate is controlled by the dissipation rate re,39,42 $^{30}$

$$
\dot{\gamma}_{\mathrm{t}}=\sqrt{\varepsilon \rho / \eta}
$$

It is worth considering whether this source of local shear can result in exfoliation. Making the approximation that all of the inputted power, $P$, is dissipated via turbulence $(i . e . \varepsilon \approx P / \rho V$ ) allows us to write $\dot{\gamma}_{\mathrm{t}} \approx \sqrt{P / V \eta}$. Then, at maximum blade speed, $P=400 \mathrm{~W}$ and if $V=0.5 \mathrm{l}, \dot{\gamma}_{\mathrm{t}} \approx 2.8 \times 10^{4} \mathrm{~s}^{-1}$, well above the threshold of $\dot{\gamma}_{\mathrm{c}} \sim 10^{4} \mathrm{~s}^{-1}$ for graphene production. In addition, this mean shear rate will be roughly constant over the entire volume of the jug meaning that graphene is produced everywhere in the jug. It is likely that this delocalisation of graphene production results in the low volume exponent discussed above. This is in contrast to rotor-stator mixers where production is probably more localised in the vicinity of the rotor resulting in a considerably more negative volume exponent $(\sim-0.5) .{ }^{26}$

However, it is worth noting that local values of the energy dissipation rate can be significantly higher than the mean value. ${ }^{36,41,42}$ Such local hotspots will also display locally high values of turbulent shear rate. We note that extremely high local values of the energy dissipation rate, and so turbulent shear rate, will be very short lived. However, because the lifetime of such fluctuations will increase as the local dissipation rate decreases, we expect there to be some threshold value of energy dissipation rate, $\varepsilon_{\max }$, and so local turbulent shear rate, $\dot{\gamma}_{\max }$, such that high-rate fluctuations persist long enough to result in exfoliation. We propose that widespread exfoliation will occur so long as $\dot{\gamma}_{\max }>\dot{\gamma}_{c}$ in a reasonable portion of the liquid for a reasonable fraction of the time. However, if the input power is reduced, at some point this will no longer hold. This explains why graphene production falls off below a critical rotation rate, $N_{\mathrm{c}}$ (Fig. 3F). We can analyse this quantitatively as follows. In reality, only a fraction, $k_{1}$, of the input power, $P$, results in turbulent kinetic energy dissipation: $\varepsilon=k_{1} P / \rho V$. The rest supports periodic motion, such as large-scale rotation of the liquid. ${ }^{39}$ Then, by defining $\varepsilon_{\max }=k_{2} \varepsilon$ and assuming $\dot{\gamma}_{\max } \approx \sqrt{\varepsilon_{\max } \rho / \eta}$, we can use eqn (2) and (3) to write:

$$
\dot{\gamma}_{\max } \approx \sqrt{\frac{k_{1} k_{2} \rho}{\eta} \frac{N_{\mathrm{p}} N^{3} D^{5}}{V}}
$$

We expect $k_{1}$ to be somewhat less than 1 while $k_{2}$ could be considerable larger than 1 . In fact, for a blade type similar to ours, $k_{2} \sim 100$ (ref. 36 ), so here $k_{1} k_{2}$ should be $\sim 10$ to 100 . We expect exfoliation to stop once $\dot{\gamma}_{\max } \sim \dot{\gamma}_{\mathrm{c}}$ which manifests itself experimentally by $N=N_{\mathrm{c}}$. This allows us to write:

$$
N_{\mathrm{c}}=\left[\frac{\eta V \dot{\gamma}_{\mathrm{c}}{ }^{2}}{k_{1} k_{2} \rho N_{\mathrm{p}} D^{5}}\right]^{1 / 3}
$$

We can use eqn (1) to estimate $N_{\mathrm{p}}$ using $P=400 \mathrm{~W}$ and $N=$ $18 \mathrm{krpm}$ to be $N_{\mathrm{p}}=0.027$, reasonably close to the value of 0.1 reported previously for a similar flat blade. ${ }^{36}$ Then, taking $\dot{\gamma}_{\mathrm{c}} \sim$ $10^{4} \mathrm{~s}^{-1}, V=300 \mathrm{ml}$ (as per the $N$-dependent study) and noting from Fig. $3 \mathrm{~F}$ that $N_{\mathrm{c}}=1800 \mathrm{rpm}\left(30 \mathrm{~s}^{-1}\right)$, we can estimate $k_{1} k_{2}=$ 75. This is well within the expected range and indicates that local turbulent shear is probably the source of graphene exfoliation in rotating blade mixers such as the blender used here. 
Finally, we can use eqn (5) to consider the properties of the ideal rotating-blade mixer for large scale graphene production. Such a mixer should have as low a value of $N_{\mathrm{c}}$ as possible to minimise operating speed. Assuming exfoliation will be in an aqueous environment, the only parameters in eqn (5) which can be controlled are $V, k_{1}, k_{2}, D$ and $N_{\mathrm{p}}$. The last three parameters are associated with the blade, emphasising the importance of blade choice. We require both $k_{2}$ and $N_{\mathrm{p}}$ to be as large as possible. However, we are not aware of a blade type that fulfils this requirement. For example, the EkatoMizer blade has low $N_{\mathrm{p}}$ and high $k_{2}{ }^{36} \mathrm{~A}$ high value of $k_{1}$ is also needed to ensure as much of the input power as possible is transformed into turbulent kinetic energy rather than rotational kinetic energy. This can be achieved by optimising the baffle arrangement. ${ }^{35}$ Scaleup generally involves increasing $V$. However this will increase $N_{\mathrm{c}}$. To avoid this, $D$ must be increased in parallel. It is likely that $D$ would scale with the dimensions of the tank: $D \propto$ $V^{1 / 3}$. In this case, $N_{\mathrm{c}} \propto V^{-2 / 9}$, resulting in a weak reduction in $N_{\mathrm{c}}$ as the volume is increased.

\section{Conclusion}

In conclusion, we have demonstrated that graphite can be exfoliated to give high-quality graphene using a very simple form of rotating-blade mixer: a kitchen blender. Moreover, sophisticated surfactants are not necessary to stabilise the exfoliated graphene, simple kitchen detergent works extremely well. We find the concentration of exfoliated graphene increases linearly with time resulting in a time-independent production rate. In addition, concentrations of $\sim 1 \mathrm{mg} \mathrm{ml}^{-1}$ were achieved, far higher than with rotor-stator mixers and comparable to what can be achieved with sonication. Importantly, the concentration decays very weakly as the volume was increased resulting in a production rate that increased with volume, a property that is critical for scale-up. We suggest that exfoliation is enabled by the locally high shear rates associated with high Reynolds number turbulence. Finally, we note that while we focus on the production of graphene using this method, it can easily be extended to exfoliate inorganic layered compounds such as $\mathrm{BN}, \mathrm{MoS}_{2}$ or $\mathrm{MoO}_{3} \cdot{ }^{32,43-45}$ It is important to note that the value of this work is not the demonstration that kitchen blenders can be used to produce graphene, although this may be a useful result. Rather, this work shows that industrial rotating blade stirred tank reactors are a promising new technology for large scale graphene production.

\section{Materials and methods}

\section{Dispersion preparation and characterization}

Flake graphite (size +100 mesh) was purchased from Sigma Aldrich (Product no. 332461) and used as supplied. The surfactant used was Fairy washing-up liquid (commonly known as Fairy Liquid, FL), a common household dishwashing liquid, with a composition of $15-30 \%$ anionic surfactants, $5-15 \%$ nonionic surfactants (specified by the manufacturer, Proctor \& Gamble). In this study, we used a Kenwood kitchen blender (model BL 370) to disperse and exfoliate graphite in aqueous-FL solutions. To perform each mix we first dissolved the required amount of FL in deionized water by stirring. Graphite was added to the tapered blender jug and FL-water solution poured in on top. The blender was operated at full speed for the required time (where required, the speed was controlled separately, see $\mathrm{ESI}_{\dagger}^{\dagger}$ ). Such blenders are not designed for continuous operation at high speeds for long times due to excess heating. To counter this, the mixer was turned off for one minute after every minute of mixing ( 1 min on/ 1 min off duty cycle). The jug was kept in an ice bath during the off cycles. During and after mixing, aliquots of the resultant dispersions were collected (10 $\mathrm{ml}$ ) and centrifuged (Hettich, Mikro 22R with fixed angle rotor) at $1500 \mathrm{rpm}(\mathrm{RCF}=231)$ for $45 \mathrm{~min}$. The top $5 \mathrm{ml}$ of each dispersion was retained for analysis. The concentration was measured using UV-vis extinction spectrometry (Varian Cary $6000 \mathrm{i}$ ) in the wavelength range of 400-800 $\mathrm{nm}$. The extinction was recorded at $660 \mathrm{~nm}$ using $10 \mathrm{~mm}$ glass cuvettes, and an extinction coefficient $\alpha_{660}=6600\left(\mathrm{mg} \mathrm{ml}^{-1}\right)^{-1} \mathrm{~m}^{-1}$ as measured previously. ${ }^{19}$ N.B. this value is larger than sometimes reported. This means the reported concentrations may be slightly underestimated. We assessed the error in concentration by measuring the concentration of 5 dispersions produced in the same way, finding a variation of $\sim 5 \%$.

In this way we performed a range of mixing experiments varying a range of parameters: FL concentration $\left(C_{\mathrm{FL}}\right)$, liquid volume $(V)$, blade speed $(N)$, initial graphite concentration $\left(C_{\mathrm{i}}\right)$ and mixing time $(t)$. Note the blender had to be modified to control and record the blade speed (see SI $\dagger$ ). By monitoring over time, we found the blade speed to be remarkably stable.

To compare with the mass of graphene produced by the blender with that given by sonication, we sonicated graphite in aqueous FL solution using a $750 \mathrm{~W}$ Vibracell sonicator (Sonics Inc.) fitted with a horn probe, operating at $40 \%$ amplitude. The sample volume was $220 \mathrm{ml}$ with an initial graphite concentration of $50 \mathrm{mg} \mathrm{ml}^{-1}$ and FL concentration of $6.25 \mathrm{mg} \mathrm{ml}^{-1}$. Aliquots were taken at regular intervals and centrifuged as described above.

Preliminary measurements showed the blender-exfoliated dispersions to contain $\sim 15$ wt $\%$ very small flakes. These were removed prior to TEM, AFM and Raman characterisation. To do this, a precentrifuged standard graphene-FL sample $\left(C_{\mathrm{i}}=20\right.$ $\mathrm{mg} \mathrm{ml} \mathrm{m}^{-1}, t=60 \mathrm{~min}$ ) was centrifuged at $7.5 \mathrm{krpm}$ for $90 \mathrm{~min}$. The supernatant containing the small graphene flakes was extracted and subjected to extinction spectroscopy to measure the mass percentage of small graphene flakes ( $15 \mathrm{wt} \%)$. The sediment was then redispersed (shaking by hand) in an equal volume of Millipore water and analysed as described below. We note that this technique also facilitates imaging by microscopy as most of free surfactant-FL is removed.

Transmission electron microscopy (TEM) was carried out in a JEOL 2100 microscope with a $\mathrm{LaB}_{6}$ filament, operating at 200 $\mathrm{kV}$ in bright-field TEM mode. Samples were prepared by dropcasting the dispersions onto holey carbon grids and drying overnight at $60{ }^{\circ} \mathrm{C}$ under mild vacuum.

Atomic force microscopy (AFM) was carried out on a Veeco Nanoscope-IIIa (Digital Instruments) system equipped with a Ehead (13 $\mu \mathrm{m}$ scanner) in tapping mode after depositing a drop 
of the dispersion $(10 \mu \mathrm{l}$, diluted by factor of 100) on a pre-heated $\left(150{ }^{\circ} \mathrm{C}\right) \mathrm{Si} / \mathrm{SiO}_{2}$ wafer with an oxide layer of $300 \mathrm{~nm}$. Typical image sizes were $2.5-5 \mu \mathrm{m}$ at scan rates of $0.4-0.6 \mathrm{~Hz}$. The setpoint was chosen to be as high as possible. However, it was impossible to remove all of the FL. This is problematic for AFM height measurements as the presence of both tip-surfactant and tip surface interactions tend to shift the measured nanosheet heights away from the true heights. To address this, we used the method first described by Paton et al. which calibrates the monolayer height using step height analysis. ${ }^{26,29}$

Raman spectra were acquired on a Horiba Scientific LabRam HR after drop casting $60 \mu \mathrm{l}$ of the dispersion in total in $10 \mu \mathrm{l}$ aliquots on preheated $\left(150{ }^{\circ} \mathrm{C}\right) \mathrm{Si} / \mathrm{SiO}_{2}$ wafers (300 $\mathrm{nm}$ oxide). Areas showing thick deposits from the optical micrographs were chosen. A $20 \mu \mathrm{m} \times 20 \mu \mathrm{m}$ area was mapped in $2 \mu \mathrm{m}$ steps (total of 100 spectra) with an excitation wavelength of $633 \mathrm{~nm}$. The Raman emission was collected by a $100 \times$ objective (N.A. $=$ 0.8 ) and dispersed by 600 lines per mm gratings with $10 \%$ of the incoming laser intensity. Prior to acquisition, the instrument was calibrated to the $\mathrm{G}$ band of HOPG $\left(1582 \mathrm{~cm}^{-1}\right)$.

The amount of surfactant residues remaining in the films was measured using thermogravimetric analysis (TGA). A small amount of the graphene film $(\sim 5 \mathrm{mg})$ was removed from the membrane and heated at $10{ }^{\circ} \mathrm{C} \min ^{-1}$ in air using a Perkin Elmer Pyris 1.

\section{Acknowledgements}

We acknowledge Science Foundation Ireland (11/PI/1087), the European Research Council (SEMANTICS and POC grant UP2DM), the Graphene Flagship Project $\left(\mathrm{n}^{\circ} 604391\right)$ and Thomas Swan \& Co. Ltd. for financial support. We acknowledge the Dublin Institute of Technology FOCAS centre for help with some of the Raman measurements.

\section{References}

1 A. K. Geim, Science, 2009, 324, 1530.

2 A. K. Geim and K. S. Novoselov, Nat. Mater., 2007, 6, 183.

3 K. S. Novoselov, V. I. Fal'ko, L. Colombo, P. R. Gellert, M. G. Schwab and K. Kim, Nature, 2012, 490, 192.

4 V. Nicolosi, M. Chhowalla, M. G. Kanatzidis, M. S. Strano and J. N. Coleman, Science, 2013, 340, 1420.

5 D. J. Finn, M. Lotya, G. Cunningham, R. J. Smith, D. McCloskey, J. F. Donegan and J. N. Coleman, J. Mater. Chem. C, 2014, 2, 925.

6 F. Torrisi, T. Hasan, W. P. Wu, Z. P. Sun, A. Lombardo, T. S. Kulmala, G. W. Hsieh, S. J. Jung, F. Bonaccorso, P. J. Paul, D. P. Chu and A. C. Ferrari, ACS Nano, 2012, 6, 2992.

7 B. Mendoza-Sanchez, B. Rasche, V. Nicolosi and P. S. Grant, Carbon, 2013, 52, 337.

8 V. H. Pham, T. V. Cuong, S. H. Hur, E. W. Shin, J. S. Kim, J. S. Chung and E. J. Kim, Carbon, 2010, 48, 1945.

9 P. Blake, P. D. Brimicombe, R. R. Nair, T. J. Booth, D. Jiang, F. Schedin, L. A. Ponomarenko, S. V. Morozov, H. F. Gleeson,
E. W. Hill, A. K. Geim and K. S. Novoselov, Nano Lett., 2008, 8, 1704.

10 A. B. Bourlinos, V. Georgakilas, R. Zboril, T. A. Steriotis and A. K. Stubos, Small, 2009, 52, 1841.

11 S. Barwich, U. Khan and J. N. Coleman, J. Phys. Chem. C, 2013, 117, 19212.

12 C. Eun-Young, C. Won San, L. Young Boo and N. YongYoung, Nanotechnology, 2011, 22, 365601.

13 Y. Hernandez, M. Lotya, D. Rickard, S. D. Bergin and J. N. Coleman, Langmuir, 2010, 26, 3208.

14 Y. Hernandez, V. Nicolosi, M. Lotya, F. M. Blighe, Z. Y. Sun, S. De, I. T. McGovern, B. Holland, M. Byrne, Y. K. Gun'ko, J. J. Boland, P. Niraj, G. Duesberg, S. Krishnamurthy, R. Goodhue, J. Hutchison, V. Scardaci, A. C. Ferrari and J. N. Coleman, Nat. Nanotechnol., 2008, 3, 563.

15 U. Khan, A. O'Neill, M. Lotya, S. De and J. N. Coleman, Small, 2010, 6, 864.

16 S. Y. Oh, S. H. Kim, Y. S. Chi and T. J. Kang, Appl. Surf. Sci., 2012, 258, 8837.

17 J. M. Englert, J. Röhrl, C. D. Schmidt, R. Graupner, M. Hundhausen, F. Hauke and A. Hirsch, Adv. Mater., 2009, 21, 4265.

18 M. S. Kang, K. T. Kim, J. U. Lee and W. H. Jo, J. Mater. Chem. C, 2013, 1, 1870.

19 M. Lotya, Y. Hernandez, P. J. King, R. J. Smith, V. Nicolosi, L. S. Karlsson, F. M. Blighe, S. De, Z. M. Wang, I. T. McGovern, G. S. Duesberg and J. N. Coleman, J. Am. Chem. Soc., 2009, 131, 3611.

20 M. Lotya, P. J. King, U. Khan, S. De and J. N. Coleman, ACS Nano, 2010, 4, 3155.

21 R. J. Smith, M. Lotya and J. N. Coleman, New J. Phys., 2010, 12, 125008.

22 S. Vadukumpully, J. Paul and S. Valiyaveettil, Carbon, 2009, 47, 3288.

23 M. Buzaglo, M. Shtein, S. Kober, R. Lovrincic, A. Vilan and O. Regev, Phys. Chem. Chem. Phys., 2013, 15, 4428.

24 U. Khan, A. O'Neill, H. Porwal, P. May, K. Nawaz and J. N. Coleman, Carbon, 2012, 50, 470.

25 A. O'Neill, U. Khan, P. N. Nirmalraj, J. Boland and J. N. Coleman, J. Phys. Chem. C, 2011, 115, 5422.

26 K. R. Paton, E. Varrla, C. Backes, R. J. Smith, U. Khan, A. O'Neill, C. Boland, M. Lotya, O. M. Istrate, P. King, T. Higgins, S. Barwich, P. May, P. Puczkarski, I. Ahmed, M. Moebius, H. Pettersson, E. Long, J. Coelho, S. E. O'Brien, E. K. McGuire, B. Mendoza-Sanchez, G. S. Duesberg, N. McEvoy, T. J. Pennycook, C. Downing, A. Crossley, V. Nicolosi and J. N. Coleman, Nat. Mater., 2014, 13, 624.

27 X. J. Chen, J. F. Dobson and C. L. Raston, Chem. Commun., 2012, 48, 3703.

$28 \mathrm{M}$. Yi and Z. Shen, Carbon, 2014, DOI: 10.1016/ j.carbon.2014.07.035.

29 C. Backes, R. J. Smith, N. McEvoy, N. C. Berner, D. McCloskey, H. C. Nerl, A. Arlene O'Neill, P. J. King, T. Higgins, D. Hanlon, N. Scheuschner, J. Maultzsch, L. Houben, G. S. Duesberg, J. F. Donegan, V. Nicolosi and J. N. Coleman, Nat. Commun., 2014, 5, 4576. 
30 D. Li, M. B. Muller, S. Gilje, R. B. Kaner and G. G. Wallace, Nat. Nanotechnol., 2008, 3, 101.

31 A. C. Ferrari, J. C. Meyer, V. Scardaci, C. Casiraghi, M. Lazzeri, F. Mauri, S. Piscanec, D. Jiang, K. S. Novoselov, S. Roth and A. K. Geim, Phys. Rev. Lett., 2006, 97.

32 D. Hanlon, C. Backes, T. M. Higgins, M. Hughes, A. O'Neill, P. J. King, N. McEvoy, G. S. Duesberg, B. Mendoza Sanchez, H. Pettersson, V. Nicolosi and J. N. Coleman, Chem. Mater., 2014, 26, 1751.

33 U. Khan, H. Porwal, A. O'Neill, K. Nawaz, P. May and J. N. Coleman, Langmuir, 2011, 27, 9077.

34 F. A. Holland, Fluid Flow for Chemical Engineers, Chemical Publishing Company, 1973.

35 F. A. Holland and F. S. Chapman, Liquid mixing and processing in stirred tanks, Reinhold Pub. Corp, 1966.

36 L. Xie, C. D. Rielly, W. Eagles and G. Ozcan-Taskin, Chem. Eng. Res. Des., 2007, 85, 676.

37 A. T. Utomo, M. Baker and A. W. Pacek, Chem. Eng. Res. Des., 2008, 86, 1397.

38 J. A. Boxall, C. A. Koh, E. D. Sloan, A. K. Sum and D. T. Wu, Langmuir, 2012, 28, 104.

39 R. Wengeler and H. Nirschl, J. Colloid Interface Sci., 2007, 306, 262.
40 A. A. Townsend, The Structure of Turbulent Shear Flow, Cambridge University Press, Cambridge, 1978.

41 S. B. Pope, Turbulent Flows, Cambridge University Press, Cambridge, 2000.

42 D. L. Marchisio, M. Soos, J. Sefcik and M. Morbidelli, AIChE J., 2006, 52, 158.

43 J. N. Coleman, M. Lotya, A. O'Neill, S. D. Bergin, P. J. King, U. Khan, K. Young, A. Gaucher, S. De, R. J. Smith, I. V. Shvets, S. K. Arora, G. Stanton, H. Y. Kim, K. Lee, G. T. Kim, G. S. Duesberg, T. Hallam, J. J. Boland, J. J. Wang, J. F. Donegan, J. C. Grunlan, G. Moriarty, A. Shmeliov, R. J. Nicholls, J. M. Perkins, E. M. Grieveson, K. Theuwissen, D. W. McComb, P. D. Nellist and V. Nicolosi, Science, 2011, 331, 568.

44 R. J. Smith, P. J. King, M. Lotya, C. Wirtz, U. Khan, S. De, A. O'Neill, G. S. Duesberg, J. C. Grunlan, G. Moriarty, J. Chen, J. Z. Wang, A. I. Minett, V. Nicolosi and J. N. Coleman, Adv. Mater., 2011, 23, 3944.

45 M. M. Y. A. Alsaif, K. Latham, M. R. Field, D. D. Yao, N. V. Medehkar, G. A. Beane, R. B. Kaner, S. P. Russo, J. Z. Ou and K. Kalantar-zadeh, Adv. Mater., 2014, 26, 3931 . 